\title{
Tantalum sound velocity under shock compression
}

Cite as: J. Appl. Phys. 125, 145903 (2019); https://doi.org/10.1063/1.5054332

Submitted: 30 August 2018. Accepted: 21 March 2019. Published Online: 11 April 2019

Minta C. Akin (D), Jeffrey H. Nguyen, Martha A. Beckwith, Ricky Chau, W. Patrick Ambrose, Oleg V. Fat'yanov (D), Paul D. Asimow (D), and Neil C. Holmes

\section{ARTICLES YOU MAY BE INTERESTED IN}

Application of $\mathrm{Al}-\mathrm{Cu}-\mathrm{W}-\mathrm{Ta}$ graded density impactors in dynamic ramp compression experiments

Journal of Applied Physics 125, 145902 (2019); https://doi.org/10.1063/1.5055398

Inferring the high-pressure strength of copper by measurement of longitudinal sound speed in a symmetric impact and release experiment

Journal of Applied Physics 125, 145901 (2019); https://doi.org/10.1063/1.5068730

Pressure-induced reentrant structural transition and equation of state of indium

Journal of Applied Physics 125, 075901 (2019); https://doi.org/10.1063/1.5086318

\section{Applied Physics Reviews} Now accepting original research

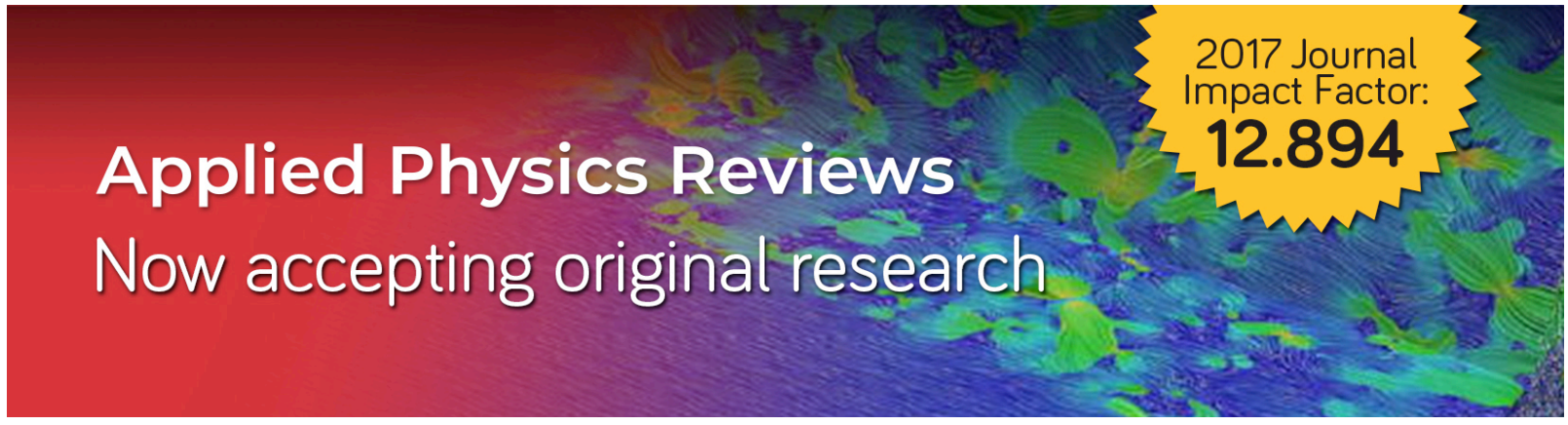




\title{
Tantalum sound velocity under shock compression
}

\author{
Cite as: J. Appl. Phys. 125, 145903 (2019); doi: 10.1063/1.5054332 \\ Submitted: 30 August 2018 Accepted: 21 March 2019. \\ Published Online: 11 April 2019
}

\begin{abstract}
Minta C. Akin, ${ }^{1, a)}$ (D) Jeffrey H. Nguyen, ${ }^{7}$ Martha A. Beckwith, ${ }^{1, b)}$ Ricky Chau, ${ }^{7}$ W. Patrick Ambrose, Oleg V. Fat'yanov, ${ }^{2}$ (D) Paul D. Asimow, ${ }^{2}$ iD and Neil C. Holmes ${ }^{1}$
\end{abstract}

\author{
AFFILIATIONS \\ 'Lawrence Livermore National Laboratory, Livermore, California 94550, USA \\ ${ }^{2}$ California Institute of Technology, Pasadena, California 91125, USA \\ a)akin1@llnl.gov \\ ${ }^{\text {b) }}$ Present address: Structural Biology Initiative, CUNY Advanced Science Research Center, New York, New York 10031, USA.
}

\begin{abstract}
We used several variations of the shock compression method to measure the longitudinal sound velocity of shocked tantalum over the pressure range $37-363 \mathrm{GPa}$ with a typical uncertainty of $1.0 \%$. These data are consistent with Ta remaining in the bcc phase along the principal Hugoniot from ambient pressure to $\approx 300 \mathrm{GPa}$, at which pressure melting occurs. These data also do not support the putative melting phenomena reported below $100 \mathrm{GPa}$ in some static compression experiments.
\end{abstract}

\section{Published under license by AIP Publishing. https://doi.org/10.1063/1.5054332}

\section{INTRODUCTION}

Tantalum, for reasons of its simple and stable crystalline structure, high density, and chemical stability, is one of the candidates for a high pressure standard. There are nevertheless, disagreements on its physical properties such as high pressure sound velocity, the melt line, existence of the $\omega$-phase, ${ }^{1}$ and low pressure phase transitions. Many of these questions can be directly or indirectly addressed by high-pressure measurements of the longitudinal sound velocity on the principal Hugoniot.

The bcc transition metals, including Ta, Mo, W, and V, have been observed to have "flat" (that is, nearly isothermal above a certain pressure) melting curves in diamond anvil cell (DAC) studies." These studies include data collected from X-ray diffraction (XRD). These results are controversial and are inconsistent with melting curves that one would expect using the Lindemann melt criterion. ${ }^{2}$ Other DAC experiments using similar techniques ${ }^{5}$ give quite different results and appear consistent with Lindemann-like behavior.

Dynamic experiments such as shock compression in two-stage light gas guns can produce stress-density data at better than $0.5 \%$ uncertainty. ${ }^{6}$ For this reason, Hugoniot data for tantalum and platinum ${ }^{7}$ have been used to derive pressure standards for $\sim 100 \mathrm{GPa}$ experiments for the past few decades. For use as a pressure standard, though, it is essential to know the phase to which the obtained equation of state data pertains. Unfortunately, dynamic phase transitions such as melting in metals, including Fe, Mo, Sn, and $\mathrm{Ta}$, are difficult to discern through changes in slope or discontinuities in either stress-density $(\mathrm{P}-\rho)$ or shock velocity-particle velocity $\left(U_{s}-U_{p}\right)$ along the Hugoniot. ${ }^{7-9}$ Certainly, no such changes are resolved in the currently available $\mathrm{Ta} U_{s}-U_{p}$ data, which are remarkably linear. ${ }^{6,7}$ A closer examination of $U_{p}$ vs time $t$ may yield subtle signs of phase transitions. ${ }^{10,11}$

Sound speed experiments can yield a much clearer signature of a phase transition, especially for melting. The discontinuous changes in longitudinal sound speed $\left(C_{L}\right)$ in such experiments can occur either because of shear-softening solid-solid transitions or because the shear modulus $G$ approaches zero upon melting. Structurally determined adiabatic bulk $(K)$ and shear moduli likewise manifest a discontinuity in sound speed in a solid-solid phase transition, as the sound speed is related to these moduli through

$$
\rho C_{L}^{2}=K+\frac{4 G}{3},
$$

where $\rho$ is the density. ${ }^{12}$ Many sound speed experiments, examining a variety of materials, have been carried out on the Hugoniot for this purpose. ${ }^{13-16}$ However, there have been discrepancies among Hugoniot sound speed studies of iron and molybdenum concerning the existence of high pressure phases and the quantification of melting pressures, ${ }^{13-16}$ some of which may be attributable 
to the use of $\mathrm{Ta}$ as a reference material in some studies. To reconcile both the Mo phase transition issue and the discrepancies among reported melting pressures of $\mathrm{Ta}$, a new high-precision campaign of Ta sound speed measurements covering the span of reported melting pressure is needed. ${ }^{13}$

At lower pressures on the Hugoniot, well before any claimed melting points, there are discrepancies in the existence of a phase transition near $60 \mathrm{GPa}$ between the findings of $\mathrm{Hu}$ et al., ${ }^{17}$ and $\mathrm{Xi}$ et al. ${ }^{18}$ To complicate the picture even further, Rigg et al. ${ }^{19}$ demonstrate that an apparent transition at $\sim 51 \mathrm{GPa}$, the elasticplastic overtake pressure of $\mathrm{Ta}$, can be made to appear through a choice of the analytical method and selection of the correction factor for the shocked window. ${ }^{19}$ These studies ${ }^{17-19}$ rely heavily on the accurate measurement of particle velocity in front-surface overtake (FSO) experiments. A more accurate sound velocity measurement method and analysis is needed to resolve these differences. ${ }^{14,15,20}$

\section{METHODS}

\section{A. Experimental methods}

The experiments described in this study rely upon the rear-surface overtake (RSO) method, which has been described extensively elsewhere. ${ }^{13-16}$ Our focus here is to get the most accurate sound velocity data with minimum uncertainty. This requires a very precise measurement of the sample and impactor thicknesses. Flatness and parallelism on the surfaces are kept to $\sim 2 \mu \mathrm{m}$ (see Table I). In our experiments, $0.8-\mathrm{mm}$ thick Ta impactors were launched into Ta targets using the propellant and light gas guns at velocities ranging from 1.1 to $6.03 \mathrm{~km} / \mathrm{s}$. We used the same target design and analysis as described in Nguyen et al. ${ }^{14}$ We used symmetric impact in all experiments, where both the target and the impactor are made of the same material, to minimize the overall experimental errors and dependence on properties of materials other than Ta. Ta samples and impactors were $99.98 \%$ pure polycrystalline materials and were sourced from legacy LLNL material

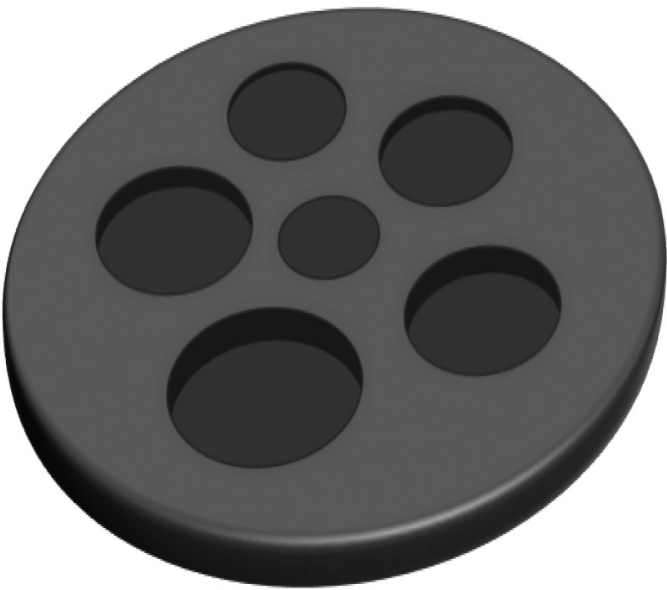

FIG. 1. Target design for the Ta baseplates used in this series. Each "pocket" cut in the baseplate creates a uniform step thickness. This allows six thicknesses to be studied per shot and improves uncertainties by correcting for reflections at the downrange surface. Reproduced with permission from Nguyen et al., Phys. Rev. B 89 174109, ${ }^{14}$ Copyright 2014 American Physical Society.

and ESPI Metals, with individual sample densities ranging from 16.6 to $16.7 \mathrm{~g} / \mathrm{cm}^{3}$. Impactor velocities were determined in each experiment through dual $x$-ray flash imaging and redundantly by the laser beam breaks on the propellant gun and by magnetic loops on the light gas gun. The Ta targets incorporated six different step heights, allowing the overtake distance to be more carefully determined. Step thicknesses were created by machining countersunk "pockets" into a single uniform Ta plate (Fig. 1). Two-dimensional simulations were done a priori to maximize the number of pockets and to avoid pocket to pocket wave interactions that might increase error in the measurements.

TABLE I. Data from this study. Uncertainty in the measurement of flyer velocity is $0.005 \mathrm{~km} / \mathrm{s}$; uncertainty in flyer thickness is $2 \mu \mathrm{m}$. Calculated shock pressures and densities use an initial Ta density of $16.67 \mathrm{~g} / \mathrm{cm}^{3}$.

\begin{tabular}{|c|c|c|c|c|c|c|c|c|c|}
\hline Shot & $\begin{array}{c}U_{\text {flyer }} \\
(\mathbf{k m} / \mathbf{s})\end{array}$ & $\begin{array}{c}\text { Flyer thickness } \\
(\mathbf{m m})\end{array}$ & $\begin{array}{c}\text { Flyer density } \\
\left(\mathrm{g} / \mathrm{cm}^{3}\right)\end{array}$ & $U_{s}(\mathrm{~km} / \mathrm{s})$ & $U_{p}(\mathrm{~km} / \mathrm{s})$ & $\begin{array}{c}\text { Pressure } \\
\text { (GPa) }\end{array}$ & $\begin{array}{c}\text { Density }(\rho) \\
\left(\mathrm{g} / \mathrm{cm}^{3}\right)\end{array}$ & $\begin{array}{c}\text { Catchup } \\
\text { distance }(\mathbf{m m})\end{array}$ & $C_{L}(\mathrm{~km} / \mathrm{s})$ \\
\hline 1082 & 1.100 & 0.785 & 16.69 & $4.01(0.02)$ & $0.550(0.003)$ & $36.8(0.3)$ & $19.32(0.03)$ & $5.38(0.13)$ & $4.64(0.04)$ \\
\hline 1085 & 1.460 & 0.757 & 16.64 & $4.25(0.02)$ & $0.730(0.004)$ & $51.7(0.4)$ & $20.13(0.03)$ & $4.84(0.18)$ & $4.82(0.06)$ \\
\hline 1081 & 1.490 & 0.800 & 16.68 & $4.27(0.02)$ & $0.745(0.004)$ & $53.0(0.4)$ & $20.20(0.03)$ & $5.2(1.5)$ & $4.8(0.4)$ \\
\hline 1083 & 1.540 & 0.782 & 16.67 & $4.30(0.02)$ & $0.770(0.004)$ & $55.2(0.4)$ & $20.31(0.03)$ & $5.09(0.12)$ & $4.81(0.04)$ \\
\hline 1094 & 1.900 & 0.780 & 16.61 & $4.535(0.017)$ & $0.950(0.004)$ & $71.8(0.4)$ & $21.09(0.03)$ & $4.72(0.15)$ & $5.00(0.06)$ \\
\hline 1092 & 2.180 & 0.782 & 16.64 & $4.718(0.015)$ & $1.090(0.004)$ & $85.7(0.4)$ & $21.68(0.03)$ & $4.22(0.07)$ & $5.28(0.04)$ \\
\hline 1086 & 2.600 & 0.770 & 16.64 & $4.992(0.014)$ & $1.300(0.004)$ & $108.2(0.4)$ & $22.54(0.03)$ & $3.81(0.12)$ & $5.56(0.08)$ \\
\hline 446 & 3.533 & 0.800 & 16.69 & $5.60(0.011)$ & $1.766(0.004)$ & $165.0(0.5)$ & $24.35(0.03)$ & $3.37(0.06)$ & $6.22(0.06)$ \\
\hline 445 & 3.870 & 0.800 & 16.67 & $5.822(0.011)$ & $1.935(0.004)$ & $187.8(0.5)$ & $24.97(0.03)$ & $3.15(0.05)$ & $6.53(0.06)$ \\
\hline 444 & 4.449 & 0.800 & 16.68 & $6.200(0.010)$ & $2.224(0.004)$ & $229.9(0.5)$ & $26.00(0.03)$ & $3.07(0.04)$ & $6.78(0.06)$ \\
\hline 447 & 5.017 & 0.800 & 16.68 & $6.572(0.009)$ & $2.509(0.004)$ & $274.8(0.5)$ & $26.96(0.03)$ & $3.26(0.02)$ & $6.71(0.03)$ \\
\hline 452 & 5.100 & 0.800 & 16.67 & $6.626(0.009)$ & $2.550(0.004)$ & $281.7(0.6)$ & $27.10(0.03)$ & $3.26(0.06)$ & $6.73(0.07)$ \\
\hline 448 & 5.386 & 0.800 & 16.64 & $6.813(0.009)$ & $2.693(0.004)$ & $305.8(0.6)$ & $27.57(0.03)$ & $3.67(0.07)$ & $6.41(0.06)$ \\
\hline 449 & 6.027 & 0.800 & 16.67 & $7.232(0.008)$ & $3.014(0.004)$ & $363.3(0.6)$ & $28.58(0.03)$ & $3.52(0.08)$ & $6.70(0.07)$ \\
\hline
\end{tabular}


Two detection methods were used. The first method uses bromoform $\left(\mathrm{CHBr}_{3}\right)$ as an analyzer. This system has been described in detail in previous papers, ${ }^{14,15}$ so we will include only a short description here. The target is enclosed in a vacuum-tight chamber, which is filled with degassed bromoform. Upon impact, twin shocks are launched, downrange into the target, and uprange into the impactor. When the downrange shock front enters the bromoform, it emits brightly, with the response scaling as $U_{p}^{7.6} \cdot{ }^{21}$ The emitted light is optically relayed to photomultiplier tubes with a large linear dynamic range, which serve as the detector system. The voltage output from the photomultipliers is recorded on fast oscilloscopes. When the uprange shock reaches the rear surface of the impactor, now moving at $U_{p}$, it launches a release wave that moves downrange in the impactor at $C_{L}+U_{p}$, a velocity which always exceeds $U_{s}$. This release wave moves through the compressed sample to eventually catch up to the shock front in the bromoform. When the release and shock fronts interact, the shock pressure and $U_{p}$ decrease, leading to a decrease in emission. We identify this catchup point as the intercept of two linear segments through a Monte Carlo algorithm described elsewhere. ${ }^{20}$ The goal is to identify, by linear extrapolation, the sample thickness at which shock and rarefaction would arrive at the sample/bromoform interface simultaneously, a result which is independent of multiple wave interactions in the sample or wave speeds in the bromoform.

Below $30 \mathrm{GPa}$ in bromoform, the emitted light intensity falls below the detection threshold of our photomultiplier tubes. For experiments in this pressure range $(<110 \mathrm{GPa}$ in $\mathrm{Ta})$, we use our second method, photon Doppler velocimetry (PDV). ${ }^{22}$ In this variation, the direct measurement of the particle velocity indicates when the release wave has arrived at the free surface, indicated by a drop in $U_{p}$ (Fig. 2). Catchup point identification and subsequent analysis is the same as with the analyzer-based methods. While adequate, it is less sensitive to velocity changes, as it requires a Fourier Transform analysis of data over a finite time interval and because the signal scales only linearly with $U_{p}$. As a result, the

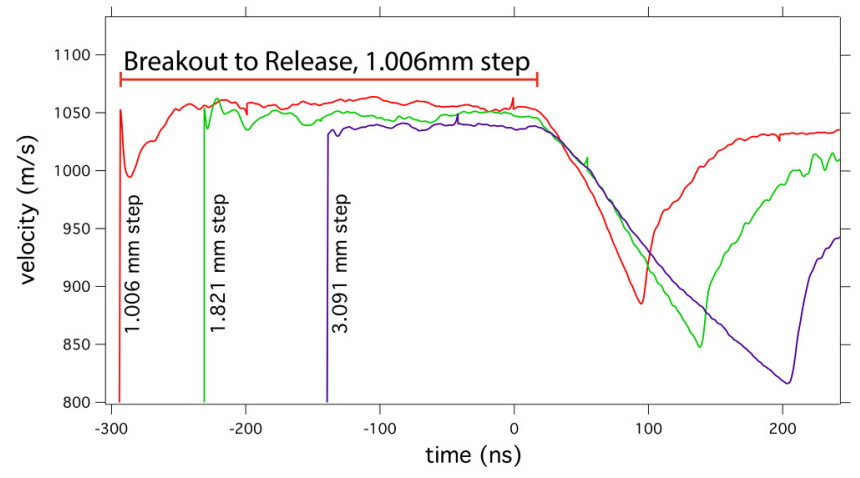

FIG. 2. Observed $U_{p}$ at selected step heights for shot 1082, which at $37 \mathrm{GPa}$ is below the elastic-plastic overtake pressure. The time between breakout and release used to calculate sound speed for the $1 \mathrm{~mm}$ step is indicated. Traces are aligned on the onset time of the rarefaction. identification of catchup times is less precise, as the slope changes are less distinct than when using an analyzer. This leads to larger uncertainties in the catchup time and ultimately in $C_{L}$, though it does provide additional information about the material's state through the $U_{p}$ measurements.

When using PDV as the detector method, we measured from a bare surface with no windows. By doing so, we avoid additional interactions of elastic or release waves in the window, though these interactions do still occur in the sample. Because our analysis method depends on time differences with step heights, the effects of these interactions, which are linearly dependent on thickness, can be corrected through extrapolation as they are in analyzer experiments. We also avoid the issues caused by windows, namely, the choice of correction factor and variation in the equation of state, faced by $\mathrm{Hu}$ et al., ${ }^{17} \mathrm{Xi}$ et al., ${ }^{18}$ and Rigg et al., ${ }^{19}$ and discussed in detail by Rigg et al. ${ }^{19}$ Window-related errors are the main source of uncertainty in FSO measurements, and so this can be a significant advantage of the RSO method.

We used the Mitchell and Nellis ${ }^{6}$ Ta Hugoniot to determine pressure, density, and uncertainties

$$
U_{s}=3.293+1.307 U_{p} .
$$

This is the same Hugoniot used by Brown and Shaner and Rigg et al. to enable easy comparison of the data sets. ${ }^{19,23}$ The difference between that Hugoniot and the later measurements by Holmes, et al. ${ }^{7}$ is negligible. Alternate Hugoniots $\left(\right.$ Lalle $^{24}$ ) were also tested and found to shift the measured value of the sound speed by up to $2.7 \%$, while also changing the position in $P-\rho$ space. Xi et al. used a slightly different Hugoniot $\left(U_{s}=3.310+1.296 U_{p}\right)$ based on a largely unpublished data set. ${ }^{18}$ We recalculated their data using Eq. (2) and found it had a negligible effect on their results, and so we have included their as-published data in figures for comparison.

\section{B. Methods to estimate bulk and shear moduli}

To further explore a change in material response near $60 \mathrm{GPa}$, we examined the bulk and shear moduli. In the solid phase, the measurement of $C_{L}$ does not directly determine either the bulk or shear modulus; one must assume a model for one to infer the other, and so those inferred values depend directly on model choices and assumptions. In this study, we applied two methods to calculate the bulk modulus, and determined the corresponding shear modulus from our calculations of $K$ and measurement of $C_{L}$ using Eq. (1).

The first functional form we considered for estimating $K$ is

$$
\rho C_{B}^{2}=K=K_{0}\left(\frac{\rho}{\rho_{0}}\right)^{s},
$$

where the ambient bulk modulus $K_{0}=200 \mathrm{GPa}$, the ambient density $\rho_{0}=16.67 \mathrm{~g} / \mathrm{cm}^{3}$, and $s$ is a fitting parameter. Fitting to the solid Ta bulk sound speed data of $\mathrm{Hu}$ et al., we find $s=3.1$, though we note that this model fails to match the liquid sound speed data above the melting pressure.

The second model we used to calculate $K$ and the bulk sound speed $C_{B}$ is that of McQueen et al., ${ }^{27}$ rearranged to give (as in 
Duffy and Ahrens ${ }^{12}$ )

$$
\rho C_{B}^{2}=K=\rho\left(\frac{\partial P}{\partial \rho}\right)_{H}\left[1-\frac{\gamma \rho}{2}\left(\frac{1}{\rho_{0}}-\frac{1}{\rho}\right)\right]+\frac{\gamma P_{H}}{2},
$$

where $P$ is the pressure, the subscript $H$ indicates the Hugoniot condition, and $\gamma$ is the Grüneisen parameter. Calculations ${ }^{28-32}$ show that $\gamma$ varies with density, though how it varies can differ significantly between calculations. An exhaustive comparison of these models to select one to use to calculate shear and bulk moduli is left for future work. Instead, we constrained our choices by fitting to $C_{L}$ in the melt region and at ambient; this requires $\gamma$ to be about 1 near $300 \mathrm{GPa}$ and 1.6-1.7 near ambient, which the model of Cohen and Gülseren agrees with. This model has weak thermal dependence, which is another advantage as the shock temperatures are unknown. Therefore, we approximated their model with a cubic function $\left(\gamma=1.7019-6.85002 \cdot 10^{-3} P_{H}+3.0444 \cdot 10^{-5} P_{H}^{2}-5.17558 \cdot 10^{-8} P_{H}^{3}\right.$, where $P_{H}$ is the pressure on the Hugoniot) up to $300 \mathrm{GPa}$, after which $\gamma=1$, and used that to calculate $K$ and $G$.

\section{RESULTS AND DISCUSSION}

We plot the new $C_{L}$ data for $\mathrm{Ta}$ with those of previous studies ${ }^{17-19,23,25}$ in Fig. 3. New data are listed in Table I. These new data agree with previously existing data by Brown and Shaner,
$\mathrm{Hu}$ et al., and $\mathrm{Yu}$ et al. at pressures above $60 \mathrm{GPa}$, including an observed melt transition at just under $300 \mathrm{GPa} .^{17,23,25}$ We observed that the sound speed ceased increasing at $230 \mathrm{GPa}$. This result is similar to that seen in $\mathrm{Mo}^{14}$ and consistent with predictions by $\mathrm{Wu}$ et al., ${ }^{26}$ which suggests that softening of the metal prior to melting leads to a decrease in the shear modulus.

Existing controversy regarding Ta phases on the Hugoniot focuses on pressures at or below $60 \mathrm{GPa}$, where $\mathrm{Hu}$ et al. indicated a transition based on changes in the longitudinal sound speed. ${ }^{17}$ Their data are consistent with those of previous works in the small regime that overlapped, and were the first Ta sound speed data to be reported at pressures less than $100 \mathrm{GPa}$. However, Rigg et al. recently demonstrated that these results-including both the direction and magnitude of the claimed change in sound speed depend on the mathematical analysis method used, and demonstrate that this change, when it is observed, is seen at the elastic-plastic overtake pressure of $51 \mathrm{GPa}$, remarkably close to the phase transition claimed by $\mathrm{Hu}$ et al. ${ }^{19}$ That work raised the basic question of whether the claimed transition was real, or an artifact of the method used.

Additional effort was spent to obtain data above the overtake pressure of $\mathrm{Ta}$, but below the claimed transition of $60 \mathrm{GPa}$. Three shots at $51-55 \mathrm{GPa}$ show excellent reproducibility in the determined values of $C_{L}$ (inset, Fig. 3), although shot 1081 suffered

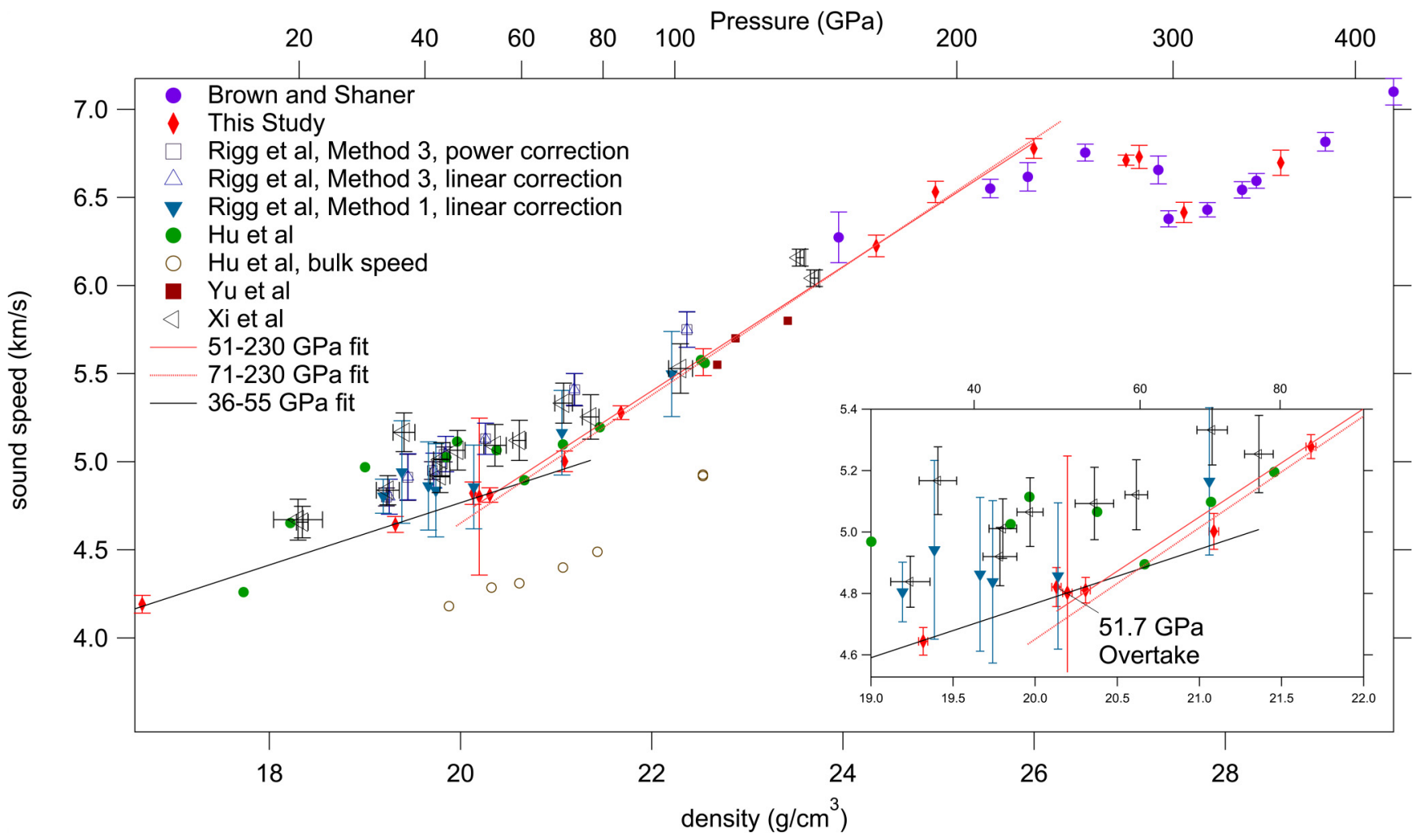

FIG. 3. The sound speed of Ta on the Hugoniot, as measured. No sharp discontinuity in sound speed is observed near $60 \mathrm{GPa}$; instead, a gradual change in the sound speed between $\sim 51$ and $72 \mathrm{GPa}$ is seen. Inset: Calculated sound speeds near the elastic-plastic overtake pressure. Error bars for other authors shown where available. 
significant data loss (two channels returned no data and a third was incomplete) resulting in larger error bars. As reproducibility studies are rarely done on sound speed measurements, we chose to include the data from shot 1081 as an illustration of both the reproducibility of the calculated sound speed, and the sensitivity of the measurement to the number and quality of data channels. There is no evidence of an elastic precursor wave in the PDV signal on any of these shots, indicating that all shots were above the elasticplastic overtake pressure. Though data are sparse, there is no discontinuity in the sound speed data between 51 and $72 \mathrm{GPa}$ comparable to that seen at $300 \mathrm{GPa}$. In fact, extrapolated fits of $C_{L}$ from 37-55 GPa, shown as a black line segment in Fig. 3, intersect $C_{L}$ at ambient pressure $(4.19 \mathrm{~km} / \mathrm{s})$ and at $72 \mathrm{GPa}(4.72 \mathrm{~km} / \mathrm{s})$ within $2 \sigma$. Similarly, fits from 72-230 GPa extrapolated to 51-55 GPa (dashed red line segment) intersect all three of the measured sound speeds in this range within $2 \sigma$. (A third fit from $51-230 \mathrm{GPa}$, shown as a solid red line segment, is included for easy comparison by the reader.) This supports the idea that the claimed transition at $60 \mathrm{GPa}$ is actually a convolution of nonideal analysis methods, window effects, and the elastic-plastic response of the material, consistent with the conclusions of Rigg et al. ${ }^{19}$ and Xi et al. ${ }^{18}$

However, extrapolation of $C_{L}$ from $51 \mathrm{GPa}$ to ambient conditions substantially underestimates the ambient sound speed, indicating an inflection point must be present. This inflection point need not be due to a phase transition. A known change in elasticplastic material response occurs near this pressure: above about $51 \mathrm{GPa}$, the plastic deformation wave overtakes the slower elastic precursor wave, combining the waves such that only a single shock is seen in the velocimetry. At pressures less than about $51 \mathrm{GPa}$, these two waves remain distinct. A single shot (1082) was obtained below the elastic-plastic overtake pressure, which shows clearly both elastic and plastic waves in the untransformed PDV traces. The emergence of the plastic wave was determined through fringe fitting, rather than the onset of any motion, which corresponded to the elastic wave. The calculated $C_{L}$ is based upon the onset of the plastic shock front, not the elastic front. This point falls directly on the extrapolation between the points at $\sim 51 \mathrm{GPa}$ and the ambient sound speed. We observed small thickness-correlated changes in the mean $U_{p}$ of each step on this shot, as shown in Fig. 2. In calculating $C_{L}$, we used the impactor velocity to determine the $P, \rho$ state of the sample, not the observed $U_{p}$ values; this is the same procedure as used on the other shots.

To further explore the behavior in this region, we calculated two models for the bulk moduli and inferred the corresponding shear moduli. The results are shown in Figs. 4 and 5. Uncertainties shown are solely due to uncertainty in the measurement of $C_{L}$ and do not include uncertainties associated with the models. As can be seen by comparing the Grüneisen and exponential models in Fig. 4, the true uncertainties are much larger. Within these uncertainties, we find that the shear modulus is approximately constant up to $72 \mathrm{GPa}$, after which it increases until softening around $250 \mathrm{GPa}$. The marked notch in $C_{L}$ at $60 \mathrm{GPa}$ observed by $\mathrm{Hu}$ is not seen.

A flat shear modulus is unusual, but not unheard of. Flattening or softening shear moduli has been predicted for $\mathrm{V}, \mathrm{Ni}$, and $\mathrm{Ta},{ }^{34}$ and was observed in the diamond anvil cell compressed $\mathrm{Ta}^{35}$ from about $50-100 \mathrm{GPa}$, though at room temperature. Differences between observed and predicted pressures for these flat

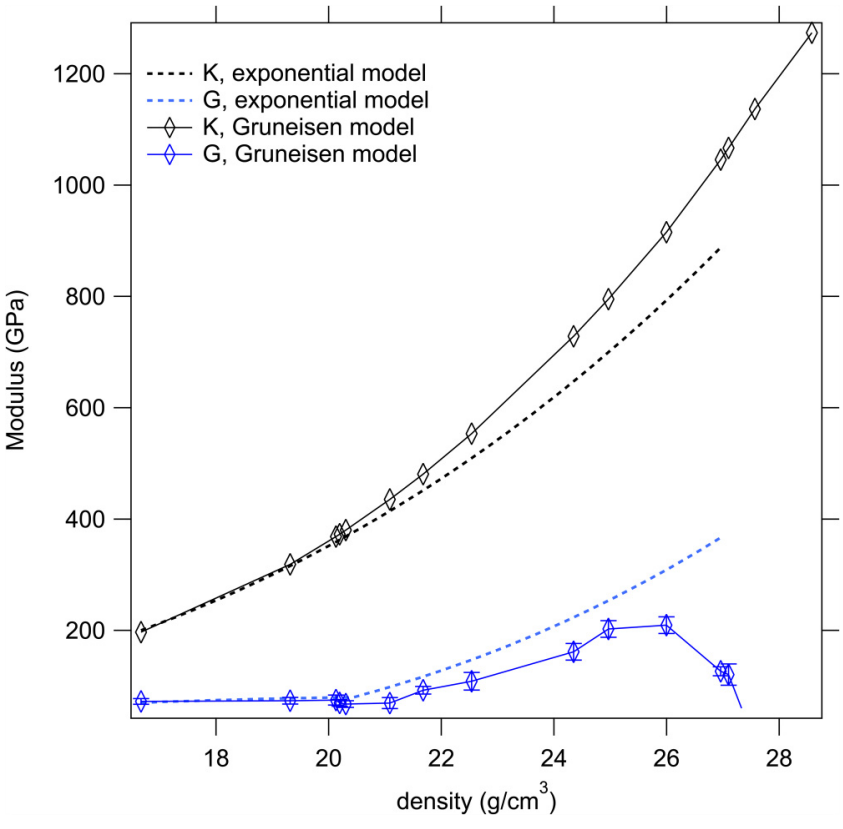

FIG. 4. Calculated bulk and shear moduli for Ta using the exponential and Grüneisen models. The shear modulus is flat within uncertainties up to about $21 \mathrm{~g} / \mathrm{cm}^{3}$ (72 GPa), after which it begins to rapidly increase until shear softening is seen near $26 \mathrm{~g} / \mathrm{cm}^{3}$. Uncertainties shown are due only to uncertainties in measurement of $C_{L}$; additional uncertainties arise from the choice of model, which are not included but can be substantial.

shear regions is likely due to uncertainties in the equilibrium volume of the DFT models used and can be substantial. Such a flat modulus (within error bars) was then observed in shock compressed $\mathrm{V}^{36}$ as well from ambient conditions to about $90 \mathrm{GPa}$; as with $\mathrm{Ta}$, there is an inflection in $C_{L}$ in this region.

There are several reasons why one must be cautious to avoid over-interpreting this result. The change in shear modulus is heavily dependent on the model choice for bulk modulus, and this apparent response in shear modulus can be altered substantially by changing the model for bulk modulus. For example, this response can be explained by altering the Grüneisen parameter model; ours was chosen only to fit the bulk data at ambient and in the melt region, as no experimental data were available in the relevant phase space. We note that neither of the chosen models for the bulk modulus match all of the $C_{B}$ data from $\mathrm{Hu}$ et al., ${ }^{17}$ which appears to have an inflection point between 80 and $100 \mathrm{GPa}$. If this point exists, then the bulk modulus must change at this condition, as it does in the $\mathrm{V}$ data. ${ }^{36} \mathrm{Ta}$ is thought to be remain bcc until melting, so one possibility could be an isostructural transition analogous to that of $\mathrm{Zr},{ }^{37}$ which again is unusual but not unheard of.

Because one can markedly alter the inferred behavior of the shear modulus by changing assumptions about the bulk modulus, we conclude that more data are needed on bulk sound speeds to improve models of the bulk modulus. Measurements of bulk moduli and sound speeds in the shock-compressed solid are 


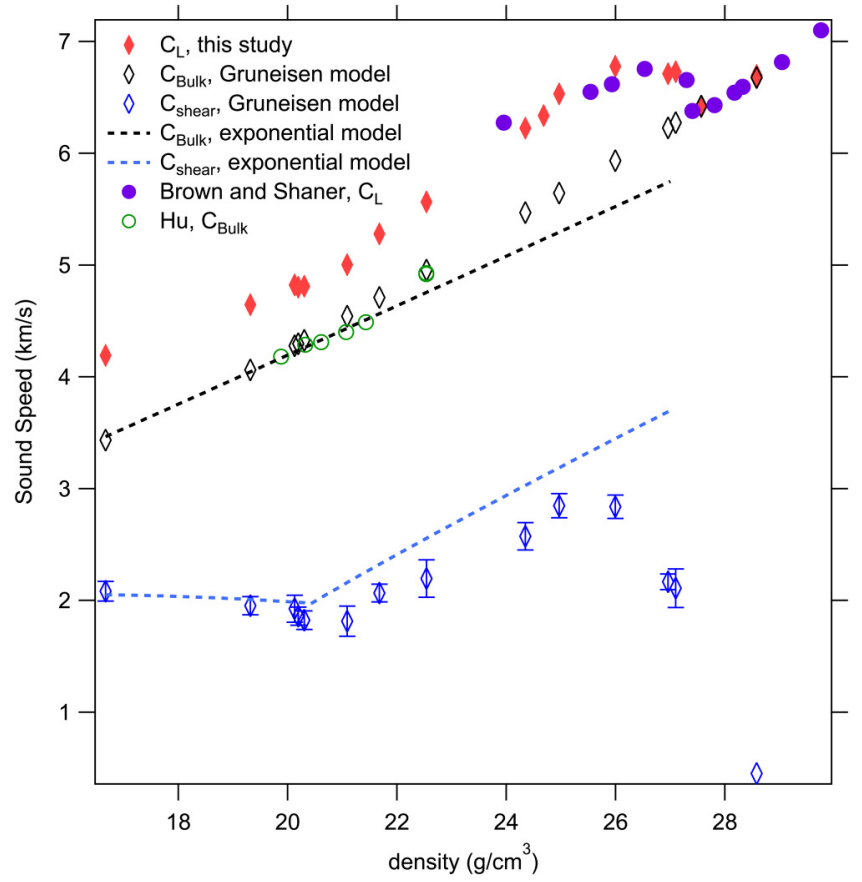

FIG. 5. Calculated bulk and shear sound speeds for Ta using the exponential and Grüneisen models. Uncertainties shown are due only to uncertainties in measurement of $C_{L}$; additional uncertainties arise from the choice of model, which are not included.

notoriously difficult, so it is reasonable to expect substantial uncertainties in these models that leave enough ambiguity in the data for either the bulk or shear moduli to change.

In light of the inferred apparently flat shear modulus, one may be tempted to dismiss these results in favor of previous work, so it is worth discussing how the data sets compare. In the studied pressure range, our data agree with the results of Rigg's Method 1. Above $51 \mathrm{GPa}$, our results also agree well with $\mathrm{Hu}$ et al.'s results, while our results are systematically lower below $51 \mathrm{GPa}^{17}$ We note a systematic $6 \%-8 \%$ discrepancy between the $C_{L}$ calculated in this study and those published by Xi et al. ${ }^{18}$ and Rigg et al. ${ }^{19}$ when using their Method 3. As Rigg et al. demonstrated, changing the method used to analyze their FSO data (to Method 1) eliminates this discrepancy. It is reasonable to expect that similar analysis changes to the Xi et al. data set would lead to similar changes.

We did not have enough information to reanalyze all of Xi et al.'s data using Rigg's Method 1, so instead, we reanalyzed $\mathrm{Xi}$ et al.'s data to test if another source of this discrepancy could be found. We note that impactor velocity was measured on only one shot in their study. However, reanalyzing their data using the impactor velocity instead of the measured $U_{p}$ had little effect. FSO is sensitive to changes in the particle velocity, including the effect of window corrections. Replacing their linear correction for particle velocity with a constant correction as described in Jensen et al. ${ }^{33}$ leads to an $\sim 1 \%$ decrease in pressure and up to a $0.5 \%$ decrease in sound speed; to approximate the differences in results observed the correction factor would have to be closer to $1.2-1.23$, which is extremely unlikely. Changing the Ta Hugoniot has even less impact (under $0.2 \%$ ) and so can be neglected. After ruling out these possibilities, three others remain: (1) sensitivities to the analytical method, as described in Rigg et al.; (2) differences arising from the technique applied, such as unaccounted-for wave interactions; and (3) differences in the material properties of the Ta used in each study. Material property differences affecting strength or shear modulus could include the grain size or the metallurgical methods used-that is, whether the samples were cast, wrought, rolled, etc. While it may be possible to test and correct for the first two reasons, if the systematic discrepancy observed is due to differences in the sample material's origin, the solution is more difficult. Unfortunately, if this is the case, measurement of a universal Ta sound speed response would not be possible, but would have to be tailored for the material at hand.

\section{CONCLUSIONS}

We measured the longitudinal sound speed of shock compressed high-purity Ta from 37 to $363 \mathrm{GPa}$ using the rear-surface overtake method. We observe a decrease in the shear modulus leading to a decreased rate of sound speed rise, consistent with previous observations, beginning near $230 \mathrm{GPa}$. A discontinuity in sound speed consistent with melting near $300 \mathrm{GPa}$, congruous with previous work, was also seen.

We find no strong evidence for the phase transition at $60 \mathrm{GPa}$ as claimed by $\mathrm{Hu}$ et al. ${ }^{17}$ Specifically, we do not observe a sharp drop in $C_{L}$ in this pressure range. Instead, the data suggest the presence of an inflection point associated with the change in the material response near $50-70 \mathrm{GPa}$; this is approximately near the elastic-plastic overtake pressure of $51 \mathrm{GPa}$ that was previously proposed by Rigg et al. ${ }^{19}$ as a possible source of the apparent transition seen by $\mathrm{Hu}$ et al. Three data points were collected at $51-55 \mathrm{GPa}$. No evidence of an elastic wave was seen, and the high agreement in $C_{L}$ demonstrates an excellent reproducibility in the method. A single point measured at $37 \mathrm{GPa}$ did show an elastic response; the calculated $C_{L}$ falls directly between $C_{L}$ at the overtake pressure and at ambient conditions, giving additional confidence in the presence of the inflection point. These results are not necessarily inconsistent with changes in the sound speed due to a single stable bcc phase up to $300 \mathrm{GPa}$, where the metal melts.

To narrow down the origin of this change in material response, the bulk and shear moduli were calculated using two different models for the bulk modulus. Doing so reveals the possible appearance of a flat shear modulus below $\sim 72 \mathrm{GPa}$, in contrast to a sharply increasing modulus as a function of pressure between $72 \mathrm{GPa}$ and $230 \mathrm{GPa}$. This flat shear modulus persists well beyond the elastic-plastic overtake condition, suggesting that some other mechanism should also be considered. As we used a Grüneisen model, an obvious possibility is some change in the thermal response or the function of $\gamma$. However, we also note that the low-pressure response is very sensitive to the model chosen for bulk modulus; a different model can easily change the apparent slope, and one must avoid assigning too much significance to the shear response until more data are available. More work should be done to experimentally extract the bulk sound speed independent of a bulk modulus 
model and the crystal phase of shock compressed Ta, especially below $\sim 70 \mathrm{GPa}$.

A systematic discrepancy was observed between the results calculated using the front-surface overtake method of Xi et al. and the rear-surface overtake method used in this study. Additional work is required to determine if these differences are due to the methods, the analysis, or the metallurgical history of the samples studied.

\section{ACKNOWLEDGMENTS}

We thank P. Rigg, P. Söderlind, and J. Klepeis for useful discussions, and the editors and reviewers for their work to improve this paper. We thank Papo Gelle, Mike Long, Russ Oliver, Mike Burns, Toni Bulai, Bob Nafzinger, Paul Benevento, Sam Weaver, and Cory McLean for their excellent and dedicated work. Lawrence Livermore National Laboratory is operated by Lawrence Livermore National Security, LLC, for the U.S. Department of Energy, National Nuclear Security Administration under Contract No. DE-AC52-07NA27344.

\section{REFERENCES}

${ }^{1}$ L. Burakovsky, S. P. Chen, D. L. Preston, A. B. Belonoshko, A. Rosengren, A. S. Mikhaylushkin, S. I. Simak, and J. A. Moriarty, Phys. Rev. Lett. 104, 255702 (2010).

${ }^{2}$ F. Lindemann, Z. Phys. 11, 609 (1910).

${ }^{3}$ D. Errandonea, B. Schwager, R. Ditz, C. Gessman, R. Boehler, and M. Ross, Phys. Rev. B 63, 132104 (2001).

${ }^{4}$ D. Santamaria-Perez, M. Ross, D. Errandonea, G. D. Mukherjee, M. Mezouar, and R. Boehler, J. Chem. Phys. 130, 124509 (2009).

${ }^{5}$ A. Dewaele, M. Mezouar, N. Guignot, and P. Loubeyre, Phys. Rev. Lett. 104, 255701 (2010).

${ }^{6}$ A. C. Mitchell and W. J. Nellis, J. Appl. Phys. 52, 3363 (1981).

${ }^{7}$ N. C. Holmes, J. A. Moriarty, G. R. Gathers, and W. J. Nellis, J. Appl. Phys. 66, 2962 (1989).

${ }^{8}$ S. P. Marsh, LASL Shock Hugoniot Data (University of California Press, 1980).

${ }^{9}$ P. Song, L. Cai, T. T. Tao, S. Yuan, H. Chen, J. Huang, X. Zhao, and X. Wang, J. Appl. Phys. 120, 195101 (2016).

${ }^{10}$ P. A. Rigg, C. W. Greeff, M. D. Knudson, G. T. Gray III, and R. S. Hixson, J. Appl. Phys. 106, 123532 (2009).

${ }^{11}$ J. Yu, W. Wang, and Q. Wu, Phys. Rev. Lett. 109, 115701 (2012).

${ }^{12}$ T. S. Duffy and T. J. Ahrens, J. Geophys. Res. 97(B4), 4503-4520 (1992).

${ }^{13}$ R. S. Hixson, D. A. Boness, J. W. Shaner, and J. A. Moriarty, Phys. Rev. Lett. 62(6), 637 (1989).
${ }^{14}$ J. H. Nguyen, M. C. Akin, R. Chau, D. E. Fratanduono, W. P. Ambrose, O. V. Fat'yanov, P. D. Asimow, and N. C. Holmes, Phys. Rev. B 89, 174109 (2014).

${ }^{15}$ J. H. Nguyen and N. C. Holmes, Nature 427, 339 (2004).

${ }^{16}$ J. M. Brown and R. G. McQueen, Geophys. Res. Lett. 7(7), 533 (1980).

${ }^{17}$ J. Hu, C. Dai, Y. Yu, Z. Liu, Y. Tan, X. Zhou, H. Tan, L. Cai, and Q. Wu, J. Appl. Phys. 111, 033511 (2012).

${ }^{18}$ F. Xi, K. Jin, L. Cai, H. Geng, Y. Tan, and J. Li, J. Appl. Phys. 117, 185901 (2015).

${ }^{19}$ P. A. Rigg, R. J. Scharff, and R. S. Hixson, J. Phys. Conf. Ser. 500, 032018 (2014).

${ }^{20}$ M. C. Akin and J. H. Nguyen, Rev. Sci. Instrum. 86(4), 043903 (2015).

${ }^{21}$ R. G. McQueen and D. G. Isassk, in Shock Waves in Condensed Matter-1989, edited by S. C. Schmidt, 1. N. Johnson, and L. W. Davison (North-Holland, Amsterdam, 1990), pp. 125-128.

${ }^{22}$ O. Strand, D. Goosman, C. Martinez, T. Whitworth, and W. Kuhlow, Rev. Sci. Instrum. 77, 083108 (2006).

${ }^{23}$ J. M. Brown and J. W. Shaner, in Shock Waves and Condensed Matter-1983, edited by J. R. Asay, R. A. Graham, and G. K. Straub (Elsevier, New York, 1984), pp. 91-94.

${ }^{24} \mathrm{P}$. Lalle and R. Courchinoux, AIP Conf. Proc. 370, 207 (1996).

${ }^{25}$ Y. Yu, H. Tan, J. Hu, C. Dai, and D. Chen, Explosion Shock Waves 26, 486 (2006).

${ }^{\mathbf{2 6}}$ C. J. Wu, P. Söderlind, J. N. Glosli, and J. E. Klepeis, Nat. Mater. 8, 223 (2009).

${ }^{27}$ R. G. McQueen, S. P. Marsh, J. W. Taylor, J. N. Fritz, and W. J. Carter, in High-Velocity Impact Phenomena, edited by R. Kinslow (Academic Press, San Diego, CA, 1970), pp. 294-419.

${ }^{28}$ C. Bercegeay and S. Bernard, Phys. Rev. B 72, 214101 (2005).

${ }^{29}$ J. A. Moriarty and J. B. Haskins, Phys. Rev. B 90, 054113 (2014).

${ }^{30}$ S. Taioli, C. Cazorla, M. J. Gillan, and D. Alfè, Phys. Rev. B 75, 214103 (2007).

${ }^{31}$ R. E. Cohen and O. Gülseren, Phys. Rev. B 63, 224101 (2001).

${ }^{32}$ Z.-L. Liu, L.-C. Cai, X.-R. Chen, Q. Wu, and F.-Q. Jing, J. Phys. Condens. Matter 21, 095408 (2009).

${ }^{33}$ B. J. Jensen, D. B. Holtkamp, P. A. Rigg, and D. H. Dolan, J. Appl. Phys. 101, 13523 (2007).

${ }^{34}$ A. Landa, P. Söderlind, A. V. Ruban, O. E. Peil, and L. Vitos, Phys. Rev. Lett. 103, 235501 (2009).

${ }^{35}$ D. Antonangeli, D. L. Farber, A. H. Said, L. R. Benedetti, C. M. Aracne, A. Landa, P. Söderlind, and J. E. Klepeis, Phys. Rev. B 82, 132101 (2010).

${ }^{36}$ Y. Yu, Y. Tan, C. Dai, X. Li, Y. Li, Q. Wu, and H. Tan, Appl. Phys. Lett. 105, 201910 (2014).

${ }^{37}$ E. Stavrou, L. H. Yang, P. Söderlind, D. Aberg, H. R. Radousky, M. R. Armstrong, J. L. Belof, M. Kunz, E. Greenberg, V. B. Prakapenka, and D. A. Young, Phys. Rev. B 98, 220101(R) (2018). 